Topological Methods in Nonlinear Analysis

Volume 46, No. 2, 2015, 967-998

DOI: $10.12775 /$ TMNA.2015.079

(C) 2015 Juliusz Schauder Centre for Nonlinear Studies

Nicolaus Copernicus University

\title{
EXISTENCE AND MULTIPLICITY OF POSITIVE SOLUTIONS FOR A SCHRÖDINGER-POISSON SYSTEM WITH A PERTURBATION
}

\author{
Juntao Sun — TSung-FAng Wu
}

\begin{abstract}
In this paper we study the nonlinear Schrödinger-Poisson system with a perturbation:

$$
\begin{cases}-\Delta u+u+K(x) \phi u=|u|^{p-2} u+\lambda f(x)|u|^{q-2} u & \text { in } \mathbb{R}^{3}, \\ -\Delta \phi=K(x) u^{2} & \text { in } \mathbb{R}^{3},\end{cases}
$$

where $K$ and $f$ are nonnegative functions, $2<q \leq p<6$ and $p>4$, and the parameter $\lambda \in \mathbb{R}$. Under some suitable assumptions on $K$ and $f$, the criteria of existence and multiplicity of positive solutions are established by means of the Lusternik-Schnirelmann category and minimax method.
\end{abstract}

2010 Mathematics Subject Classification. Primary: 35J20, 35J61; Secondary: 35A01, 35B40.

Key words and phrases. Schrödinger-Poisson systems, variational methods, LusternikSchnirelmann category, multiple solutions.

The first author was supported by the National Natural Science Foundation of China (Grant Nos. 11201270 and 11271372), Shandong Provincial Natural Science Foundation (Grant Nos. ZR2012-AQ010 and ZR2015JL002), China Postdoctoral Science Foundation (Grant Nos. 2014M551494 and 2015T80491), and Young Faculty Support Program of Shandong University of Technology. The second author was supported in part by the Ministry of Science and Technology, Taiwan (Grant 102-2115-M-390-002-MY2) and the National Center for Theoretical Sciences, Taiwan. 


\section{Introduction}

In this paper we are concerned with the coupled system of SchrödingerPoisson equations of the form:

$$
\begin{cases}-\Delta u+u+K(x) \phi(x) u=h(x, u) & \text { in } \mathbb{R}^{3} \\ -\Delta \phi=K(x) u^{2} & \text { in } \mathbb{R}^{3}\end{cases}
$$

where $K$ is a nonnegative function and $h: \mathbb{R}^{3} \times \mathbb{R} \rightarrow \mathbb{R}$ is a Carathédory function.

Such a system, also known as the nonlinear Schrödinger-Maxwell equations, have a strong physical meaning. It was first introduced in [8] as a model describing solitary waves for the nonlinear stationary Schrödinger equations interacting with the electrostatic field, and also in semiconductor theory, in nonlinear optics and in plasma physics. Indeed, in Problem (SP) the first equation is a nonlinear stationary Schrödinger equation (where, as usual, the nonlinear term simulates the interaction between many particles) that is coupled with a Poisson equation, to be satisfied by $\phi$, meaning that the potential is determined by the charge of the wave function.

In recent years, problem (SP) has been studied widely via variational methods under the various hypotheses on $K$ and $f$, see [3], [5], [6], [13], [14], [18], [20], [24], [25] and the references therein. Now we recall some of them as follows.

If $h(x, u) \equiv|u|^{p-2} u$ and $K(x) \equiv \mu>0$, Ruiz [24] gave existence and nonexistence results on positive radial solutions of problem (SP), depending on the parameters $p$ and $\mu$. It turned out that $p=3$ is a critical value for the existence of solutions. Later, the results in [24] were further improved in Ambrosetti and Ruiz [5] by showing the presence of multiple bound states when certain conditions on the parameters are satisfied.

If $h(x, u) \equiv a(x)|u|^{p-2} u$ and $K(x) \equiv \mu>0$, Chen et al. [14] studied the multiplicity of positive solutions for problem (SP) with $4 \leq p<6$. They showed that the number of positive solutions are dependent on the profile of the function $a$.

If $h(x, u) \equiv a(x)|u|^{p-2} u$ and $K$ is a nonnegative $L^{2}$-function, Cerami and Vaira [13] obtained the existence of positive ground state and bound state solutions for problem (SP) with $4<p<6$ under some suitable assumptions, but not requiring any symmetry property on $a$ and $K$.

Motivated by these findings, we now extend the analysis to the nonlinear Schrödinger-Poisson system with a perturbation. Our intension here is to illustrate the difference in the solution behavior which arises from the consideration of the perturbation. Here we consider the following Schrödinger-Poisson systems:

$$
\begin{cases}-\Delta u+u+K(x) \phi(x) u=|u|^{p-2} u+\lambda f(x)|u|^{q-2} u & \text { in } \mathbb{R}^{3} \\ -\Delta \phi=K(x) u^{2} & \text { in } \mathbb{R}^{3}\end{cases}
$$

